\title{
Neuroprotective Effects of Ginkgo biloba extract, GBB, in the Transient Ischemic Rat Model
}

\author{
Jin Kyung OH${ }^{1}$, Ji Wook Jung ${ }^{2}$, Hye Rim OH${ }^{1}$, Yong Nam Hav ${ }^{3}$ and Jong Hoon Ryu ${ }^{1,4 *}$ \\ ${ }^{1}$ Department of Oriental Pharmaceutical Science and Kyung Hee East-West Pharmaceutical Research Institute, \\ College of Pharmacy, ${ }^{4}$ Department of Department of Life and Nanopharmaceutical Science, Kyung Hee University, Korea \\ ${ }^{2}$ Department of Herbal Medicinal Resource, College of Health and Welfare, Daegu Haany University, Gyeongsan 712-715, Korea \\ ${ }^{3}$ Department of Pharmaceutical Science, College of Pharmacy, Seoul National University, Korea
}

(Received 19 July 2007; Accepted 4 September 2007)

\begin{abstract}
In the present study, we investigated the neuroprotective effects of standardized Ginkgo biloba extract (GBB) (total terpene trilactones, $13 \pm 3 \%$; biflavone, $4.5 \pm 1.5 \%$; flavonol glycoside, $<8 \%$; proanthocyanidine, under detection limit) on ischemia-reperfusion-induced brain injury in the rats. Ischemia was induced by the intraluminal occlusion of the right middle cerebral artery for $2 \mathrm{~h}$ and reperfusion was continued for $22 \mathrm{~h}$. GBB was orally administered, promptly prior to reperfusion and $2 \mathrm{~h}$ after. Total infarction volume in the ipsilateral hemispheres of ischemia-reperfusion rats were significantly reduced by treatment with GBB in a dose-dependent manner $(P<0.05)$. The therapeutic time window of GBB was $3 \mathrm{~h}$ in this ischemia-reperfusion rat model. Furthermore, GBB also significantly inhibited increased neutrophil infiltration of ischemic brain tissue, as estimated by myeloperoxidase activity. These findings suggest that GBB plays a crucial protective role in ischemia-induced brain injury, in part, via inhibition of neutrophil infiltration, and suggest that this GBB could serve as a neuroprotective agent following transient focal ischemic brain injury.
\end{abstract}

Keywords $\square$ Gingko biloba, Ischemia-reperfusion, Neutrophil, Myeloperoxidase

\section{INTRODUCTION}

Ginkgo biloba Linne (Ginkoaceae) is an ancient plant used for its medicinal properties. A standardized extract of the leaves of Ginkgo biloba (EGb 761) is widely prescribed in Europe for the treatment of cerebrovascular and neurodegenerative disorders (Weichel et al., 1999). EGb 761 contains 24\% flavonol glycosides, about $6 \%$ terpene trilactones (ginkgolides A, B, C, and bilobalides), about $7 \%$ proanthocyanidins, some organic acid, and other common compounds (van Beek, 2002). Many researches have been conducted on the role of the EGb 761 in the treatment of diseases involving free radicals and oxidative damage (DeFeudis and Drieu, 2000). EGb 761 is also well known to have antioxidative and neuroprotective properties.

Although there are several reports on the beneficial effects of EGb761, de Lima et al. (2006) reported that EGb 761 did not show any significant effect on brain infarct size in the transient

*Corresponding author

Tel: +82-2-961-9230, Fax: +82-2-966-3885

E-mail: jhryu63@khu.ac.kr middle cerebral artery occlusion (MCAO) model. Previously, we observed that proanthocyanidine contained in the EGb 761, a condensed tannin, increased the bleeding time in vitro test (unpublished data). Therefore, the extract of Ginkgo biloba without, or if any, proanthocyanidine is likely to have more effective to the ischemic brain damage. We prepared a standardized extract of the leaves of Ginkgo biloba (GBB) which is an extract of Ginkgo biloba leaves standardized for the contents of total terpene trilactones $(13 \pm 3 \%)$, biflavone $(4.5 \pm 1.5 \%)$, flavonol glycoside $(<8 \%)$, and proanthocyanidine (under detection limit) (Park et al., 2006). The characteristics of GBB compared with EGb 761 are the increase of terpene trilactones, decrease of flavonol glycoside, and no detectable proanthocyanidine.

In the present study, we investigated the effect of GBB on ischemic brain damage induced by focal ischemia-reperfusion in a rat MCAO model, and we examined its role upon neutrophil infiltration. Inflammatory cell infiltration was quantified by assaying the activity of myeloperoxidase (MPO) which is mainly located in neutrophil primary granules. 


\section{MATERIALS AND METHODS}

\section{Animals}

Male Sprague-Dawley rats, weighing 260-280 g, were purchased from the Orient Co., Ltd. of a Charles River branch (Seoul, Korea). The rats were housed 4 or 5 per cage, allowed free access to water and food, and maintained under constant temperature $\left(23 \pm 1^{\circ} \mathrm{C}\right)$ and humidity $(60 \pm 10 \%)$ under a $12 \mathrm{~h}$ light/dark cycle (light on 07.30-19.30 h). This study was conducted in accordance with the Principles of Laboratory Animal Care (NIH publication, \#85-23, revised 1985) and the Animal Care and Use guidelines of Kyung Hee University, Korea.

\section{Transient focal cerebral ischemia}

Animals were anesthetized in a chamber with a mixture of $\mathrm{N}_{2} \mathrm{O}$ and $\mathrm{O}_{2}$ (70:30) containing $2.5 \%$ isoflurane and maintained by inhalation of $2 \%$ isoflurane. The middle cerebral artery (MCA) was occluded as described by Nagasawa and Kogure (1989), with minor modifications (Oh et al., 2007). Briefly, after making a median incision in the neck skin, the right common carotid artery was exposed and a 17-mm-long 4-0 nylon thread with a rounded tip coated with silicon resin (Xantoprene, Byer Dental, Germany) was inserted from the bifurcation to the right MCA. After surgery, rats were allowed to recover from the anesthesia. Two hours after MCAO, the thread was removed to allow complete reperfusion of the ischemic area under re-anesthesia. Neurological deficits characterized by severe left-sided hemiparesis and right Horner's syndrome were used as criteria to evaluate the ischemic insult. Rats that did not exhibit behavioral deficits before reperfusion were excluded from the study. Heart rate, arterial blood oxygen saturation of arterial hemoglobin and ECG were monitored throughout the procedure (SurgiVet, USA). Body temperature was maintained at $37 \pm 0.5^{\circ} \mathrm{C}$ throughout surgery by a heating pad (Biomed S.L., Spain).

\section{Drug administration}

Rats that showed positive neurological signs $2 \mathrm{~h}$ after MCAO were randomly divided into control, 3-Methyl-1-phenyl2-pyrazolium-5-one (MCI-186, Sigma Co., USA), or GBBtreated groups. EGb 761 was used as a reference drug (gifted from SK Chemical Co., Korea). GBB is an extract of Ginkgo biloba standardized for the contents of total terpene trilactones $(13 \pm 3 \%)$, biflavone $(4.5 \pm 1.5 \%)$, flavonol glycoside $(<8 \%)$, and proanthocyanidine (under detection limit). For dose-response studies, GBB $(10,20,40$, and $80 \mathrm{mg} / \mathrm{kg})$ was dissolved in saline and orally administered, promptly prior to reperfusion and again $2 \mathrm{~h}$ later. Rats in the vehicle control group were given saline orally and rats in the positive control group were given MCI-186 (10 mg/kg) orally using the same time schedule used in the GBB-treated group. In order to evaluate the therapeutic time window of GBB, double treatments of $40 \mathrm{mg} / \mathrm{kg}$ (p.o.) with a $2 \mathrm{~h}$ interval were performed at $1 \mathrm{~h}$ and $3 \mathrm{~h}, 2 \mathrm{~h}$ and $4 \mathrm{~h}$, and $3 \mathrm{~h}$ and $5 \mathrm{~h}$ after MCA occlusion.

\section{Morphometric measurement of infarct volume and edema}

The area of cerebral infarction was identified by 2,3,5-triphenyltetrazolium chloride (TTC) staining. After reperfusion for $22 \mathrm{~h}$, animals were decapitated and their brains carefully removed. The brains were sectioned coronally with a brain slicer for rat (ASI Instruments, USA) at 2-mm intervals, starting $1 \mathrm{~mm}$ from the frontal pole. Slices were incubated for 60 min in a $2 \%$ solution of TTC at $37^{\circ} \mathrm{C}$ and fixed by immersion in $10 \%$ neutral-buffered formalin. The area of infarction $\left(\mathrm{mm}^{2}\right)$ of each section was determined using a computerized image analysis system (TDI Scope Eye 3.0), and the total lesion volume $\left(\mathrm{mm}^{3}\right)$ was calculated by summing the infarct area in each section (seven slices in all) and multiplying this by the distance between sections. Both contralateral and ipsilateral hemisphere areas were measured, and the differences between the two areas were used to calculate the edema volume.

\section{Measurement of MPO activity}

MPO activity in brain tissue was determined as an index of neutrophil accumulation, as previously described (Barone et al., 1991; Hwang et al., 2002). In brief, brains were rapidly removed and cut into two $4 \mathrm{~mm}$ thick slices $(3 \mathrm{~mm}$ to $7 \mathrm{~mm}$ and $7 \mathrm{~mm}$ to $11 \mathrm{~mm}$ from the frontal pole), and then separated into right and left hemispheres. Each sample was homogenized and centrifuged at $30,000 \mathrm{~g}\left(30 \mathrm{~min}, 4^{\circ} \mathrm{C}\right)$. The pellets suspended in $0.5 \%$ hexadeacyltrimethylammonium bromide in 50 $\mathrm{mM}$ potassium phosphate buffer $(\mathrm{pH} 6.0)$ at an original tissue wet weight to volume ratio of $1: 10$. Samples were then immediately frozen on dry ice, and three freeze/thaw cycles were performed with sonication $\left(10 \mathrm{sec}, 25^{\circ} \mathrm{C}\right)$ between cycles. After the final sonication, samples were incubated at $4^{\circ} \mathrm{C}$ for $20 \mathrm{~min}$ and centrifuged at $12,500 \mathrm{~g}\left(15 \mathrm{~min}, 4^{\circ} \mathrm{C}\right)$. The supernatant so obtained $(0.05 \mathrm{ml})$ was mixed with $0.95 \mathrm{ml}$ of potassium phosphate buffer ( $\mathrm{pH} 6.0$ ) containing $0.167 \mathrm{mg} / \mathrm{ml}$ of $O$-dianisidine and $0.0005 \%$ hydrogen peroxide. Change in absorbance at 460 $\mathrm{nm}$ was measured by using a spectrophotometer equipped with a kinetic analysis capability (JASCO, Japan). One unit of MPO 
activity was defined as the amount that degraded $1 \mu \mathrm{mol}$ of peroxide/min at $25^{\circ} \mathrm{C}$. Tissue MPO activity was calculated using human MPO (Sigma Co., USA) as a standard.

\section{Statistical analysis}

Values are expressed as mean \pm S.E.M. Data were analyzed by a one-way analysis of variance (ANOVA) followed by Duncan's test for multiple comparisons. Statistical significance was set at $P<0.05$. Student's $t$-test was used to compare MPO activity with that of the control group.

\section{RESULTS}

\section{Effects of GBB on the ischemic brain damage}

Vehicle-treated rats after $2 \mathrm{~h}$ of ischemia and $22 \mathrm{~h}$ of reperfusion showed an infarct volume of $321.92 \pm 12.94 \mathrm{~mm}^{3}$. The total infarction volumes in the ipsilateral hemisphere of ischemiareperfusion rat were significantly reduced by GBB treatment in a dose-dependent manner (Fig. 1). The infarct volume was decreased to $260.43 \pm 24.26 \mathrm{~mm}^{3}, 158.32 \pm 21.17 \mathrm{~mm}^{3}, 156.09$ $\pm 11.36 \mathrm{~mm}^{3}$, and $145.39 \pm 16.23 \mathrm{~mm}^{3}$ at doses of $10,20,40$, and $80 \mathrm{mg} / \mathrm{kg}$, respectively. Most effective dose was $40 \mathrm{mg} / \mathrm{kg}$ of GBB, thereafter we adopted $40 \mathrm{mg} / \mathrm{kg}$ of GBB for therapeutic time window. Moreover, treatment with MCI-186 (10 mg/

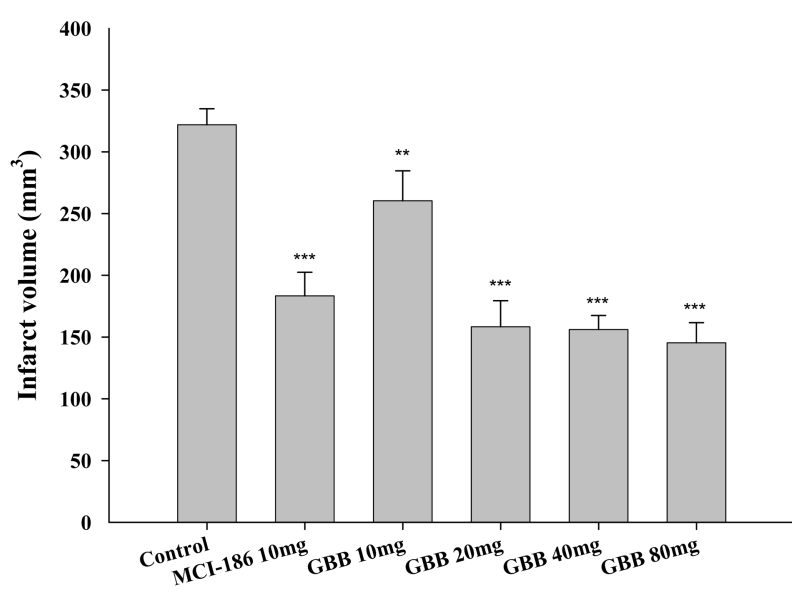

Fig. 1. Total infarct volume was measured $2 \mathrm{~h}$ after MCA occlusion followed by $22 \mathrm{~h}$ reperfusion. Vehicle $(\mathrm{n}=12)$, GBB $(10,20,40$, or $80 \mathrm{mg} / \mathrm{kg}, \mathrm{n}=8)$, and MCI-186 $(10 \mathrm{mg} / \mathrm{kg}, \mathrm{n}=8)$ were administered orally twice, promptly prior to reperfusion and $2 \mathrm{~h}$ after reperfusion. Data represent mean \pm SEM. The differences between the total infarction volumes of the control, GBB, and MCI-186 groups were determined by ANOVA followed by Duncan's test. $* * P<0.01, * * * P<0.001$ (vs. vehicletreated control group). $\mathrm{kg}$, p.o.) reduced the infarct volume by $47 \%$ versus vehicletreated control group. By the treatment of EGb 761, the infarct volume was reduced by $37 \%$ versus vehicle-treated control group (data not shown). GBB (40 mg/kg, p.o.) was administered at $1 \mathrm{~h}$ and $3 \mathrm{~h}, 2 \mathrm{~h}$ and $4 \mathrm{~h}, 3 \mathrm{~h}$ and $5 \mathrm{~h}$ after MCAO, and significantly reduced infarct volumes by $56 \%, 52 \%$, and $46 \%$, respectively compared with the control group (Fig. 2). These data indicate that the optimal therapeutic time for GBB administration was $<3 \mathrm{~h}$ in this ischemia-reperfusion rat model.

\section{Effects of GBB on the MPO activity}

Fig. 3 illustrates MPO activity in the MCA territory in response to MCAO. In the control group, MPO activity in the ipsilateral hemispheres $(0.273 \pm 0.011 \mathrm{unit} / \mathrm{g}$ wet tissue $)$ was significantly greater than that in the contralateral hemispheres. With the administration of GBB (40 mg/kg, p.o), mean MPO activity in the ipsilateral hemispheres $(0.196 \pm 0.012 \mathrm{unit} / \mathrm{g}$ wet tissue) was significantly lower than that observed in the vehicle-treated control group $(P<0.001)$.

\section{DISCUSSION}

In the present study, we found that ischemia-reperfusioninduced infarct volume was reduced by GBB in a dose-dependent manner, and that neuroprotection was still obtained even

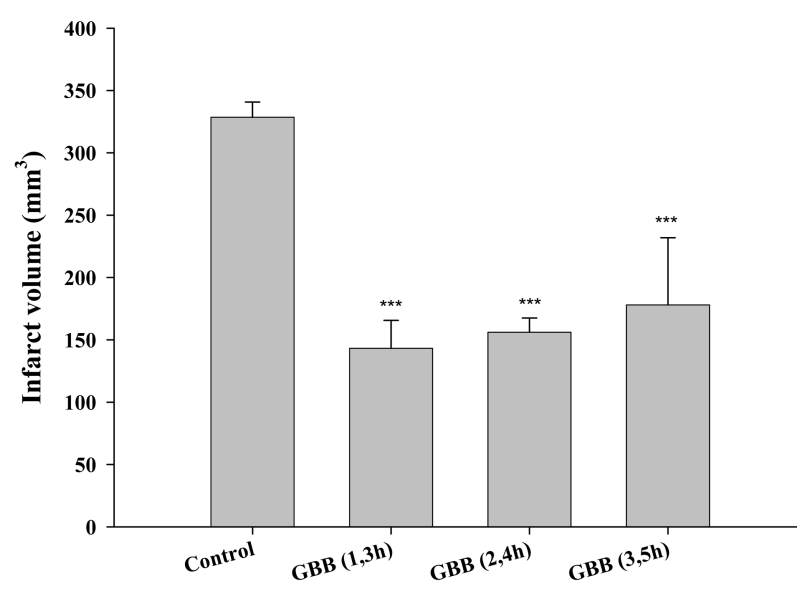

Fig. 2. In order to evaluate the therapeutic time window of the effects of the GBB, it was administered by double treatment of $40 \mathrm{mg} / \mathrm{kg}(\mathrm{n}=5)$ with a $2 \mathrm{~h}$ interval at $1 \mathrm{~h}$ and $3 \mathrm{~h}, 2 \mathrm{~h}$ and $4 \mathrm{~h}$, and $3 \mathrm{~h}$ and $5 \mathrm{~h}$ after MCA occlusion. Data represent mean \pm SEM. The differences between the total infarction volumes of the vehicle-treated control and the GBB treatment groups were determined by ANOVA followed by Duncan's test. $* * * P<$ 0.001 , (vs. vehicle-treated control group). 


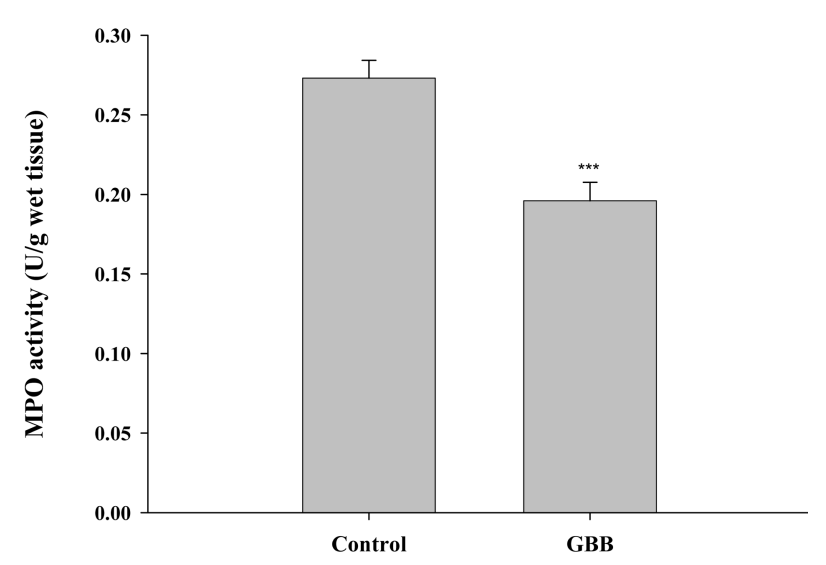

Fig. 3. MPO activity in the ipsilateral hemispheres in the GBBtreated group. Measurements were made $2 \mathrm{~h}$ after middle cerebral artery occlusion followed by $22 \mathrm{~h}$ reperfusion. Vehicle $(\mathrm{n}=8)$ and GBB (40 mg/kg, p.o., $\mathrm{n}=8)$ were administered orally twice, promptly prior to reperfusion and $2 \mathrm{~h}$ after reperfusion. Values are mean \pm SEM. $* * * P<0.001$ versus the vehicletreated control group (Student's $t$-test).

when the administration of GBB was delayed to $3 \mathrm{~h}$ after MCAO. Moreover, we observed that MPO activity was significantly suppressed in post-ischemic brain tissue after administration of GBB. Since the inhibition of MPO activity plays a critical role in injured brain tissue, it appears that the reduced brain infarct volume due to GBB treatment is more likely to be due to reduced neutrophil infiltrations.

Because free radicals play important roles in the progression of cell damage associated with ischemia, antioxidants are considered to be a promising approach to limit the extent of neuronal loss in ischemic brain injury (Fisher and Schaebitz, 2000). Edaravone (3-Methyl-1-phenyl-2-pyrazolium-5-one, MCI-186) is the case of a free radical scavenger that has been clinically used to reduce neuronal damage following cerebral ischemia since June 2001 in Japan (Tanahashi and Fukuuchi, 2002). Although edaravone exhibited marked improvement in the acute survival rate for stroke patients, chronic functional outcome among the survivors was not improved (Yoshida et al., 2006). However, almost neuroprotective agents so far targeting a specific pathway in ischemic cascade failed to demonstrate clinical efficacy (De Keyser et al., 1999). Therefore, the search for novel therapeutic approaches is even more critical. Recent report suggested a possibility that combination therapy have synergistic and will be another choice for ischemic treatment (Zhang et al., 2004). Qualified and standardized plant extract, if possible, is likely to be an example of combination therapies. A standardized extract of the leaves of Ginkgo biloba, EGb 761, has neu- roprotective and antioxidant properties. For example, oral administration of EGb 761 has been shown to reduce neuronal death in the gerbil after transient global ischemia (Calapai et al., 2000), protect against neuronal death in rats after focal ischemia induced by MCA occlusion (Lee et al., 2002). The main neuroprotective mechanism of EGb 761 is thought to be antioxidant properties. Proanthocyanidins are among the most abundant phenolic compounds in the leaves of Ginkgo biloba. Proanthocyanidins, or condensed tannins, are widely distributed in the plant kingdom and are present in a wide range of plant-derived products including Ginkgo biloba. For example, the average molecular weight of proanthocyanidins from the Hamamelis bark is reached to about $1500 \mathrm{Da}$ and $3400 \mathrm{Da}$ (Deters et al., 2001). However, there is no report on the molecular size of proanthocyanidins from Ginkgo biloba. If the molecular size of proanthocyanidins from Ginkgo biloba is above $600 \mathrm{Da}$, it is unlikely to penetrate the blood-brain barrier (BBB) because $\mathrm{BBB}$ is only permeable to lipophilic molecule of molecular weight <600 Da (Pardridge et al., 1995). We observed that the proanthocyanidin-rich fractions from Ginkgo biloba had only $10 \%$ neuroprotective properties and that proanthocyanidine contained in the EGb 761 increased the bleeding time in vitro test (data not shown). A standardized EGb 761 contains $24 \%$ flavone glycosides, about $6 \%$ terpens, about $7 \%$ proanthocyanidins, some organic acid, and other common compounds (van Beek, 2002). The flavones have a property of scavenging free radical, whereas the terpenoid fraction containing predominantly ginkgolides $\mathrm{A}$ and $\mathrm{B}$ is known to be responsible for blocking platelet aggregation in order to increase blood flow (Kudolo et al., 2003). Ginkgolide B especially has a potent activity of an anti-platelet activating factor (PAF) that causes inflammation (Zhang et al., 2000; Chung et al., 1999; Lenoir et al., 2002). The anti-inflammatory effects of the water soluble portion of EGb 761 on inflammation caused by Candida albicans revealed that the terpene, rather than the flavone portion of this material is responsible for these therapeutic effects (Han, 2005). GBB contains more terpene and lower level of flavonol glycosides than EGb 761 dose. We suggest that the terpene in GBB may be responsible for its enhanced physicochemical properties and anti-inflammatory action. As expectedly, we observed that GBB was found to have a significant neuroprotective effect after double treatment in this rat reperfusion model, approaching $52 \%$ at $22 \mathrm{~h}$ similar to those of MCI-186 used as a positive control. Furthermore, its neuroprotective effect was still observed even when treatment was delayed for $3 \mathrm{~h}$ after MCAO. Cerebral edema also occurs com- 
monly during the acute phase of a large cerebral infarction, and we found that the administration of GBB after MCAO reduced brain edema. Although, however, edema still tended to be smaller after MCAO, the reduction rates were not statistically significant (data not shown). These results suggested that GBB is a new candidate of the standardized Ginkgo biloba extracts.

Neutrophil infiltration is a hallmark of the inflammatory response in the ischemic brain damage (Barone et al., 1991). Using a MCAO model, Beray-Berthat et al. (2003) reported that neutrophils contributed to the progression of ischemic lesion in the cortical area. Moreover, administration of antiMac-1 antibodies also significantly reduced neutrophils accumulation in the ischemic cortex and subcortex (Chopp et al., 1994). In addition, Matsuo et al. (1994) observed reductions in the level of cortical and striatal neutrophils when ischemic rats were pretreated with an anti-neutrophil monoclonal antibody and they showed a positive correlation between reduced neutrophil infiltration and the subsequently reduced ischemic injury. In the present study, GBB, which reduced MPO activities by about $28 \%$, reduced total infarction volume by $52 \%$, suggesting that GBB blocked the infiltration of neurtrophil into brain tissue from blood stream (Oh et al., 2007). However, there were large differences between the MPO activity and the total infarction volume, which might be due to the presence of other protecting mechanisms triggered by GBB. Previously, we found out that GBB had inhibitory activities on the lipopolysaccharide-induced nitric oxide (NO) and prostaglandin $\mathrm{E}_{2}$ production by suppression of inducible NO synthase and cyclooxygenase-2 activities in the RAW 264.7 macrophage cell line (Park et al., 2006). In addition, Ginkgo biloba extract improved the abnormal responses and maintain the normal function of astroglial network after hypoxia/ reoxygenation, $\mathrm{H}_{2} \mathrm{O}_{2}$ or high concentration of L-glutamate injury ( $\mathrm{Li}$ et al., 2005). If GBB has similar properties in the ischemic conditions, it can be explained why MPO activity in the ischemic regions was lower than the rate of reduced infarct volume. However, the other mechanism including free radical scavenging activity of GBB can not be excluded because GBB contains several flavonoid compounds in high amounts. Moreover, MPO activity can not be fully statement for the infiltration of neutrophil because infiltrated neutrophil can be engulfed by macrophages even at earlier time points (Weston et al., 2007). Further research should be needed to clarify these issues.

In conclusion, the present study demonstrates that GBB has a neuroprotective effect on ischemia-reperfusion induced brain injury, and that its anti-ischemic effect is partly mediated via a reduction of neutrophil infiltration into brain tissue.

\section{ACKNOWLEDGEMENTS}

This research was supported by a grant funded by Seoul Research and Business Development Program (10524).

\section{REFERENCES}

Barone, F. C., Hillegass, L. M., Price, W. J., White, R. F., Lee, E. V., Feuerstein, G. Z., Sarau, H. M., Clark, R. K. and Griswold, D. E. (1991). Polymorphonuclear leukocyte infiltration into cerebral focal ischemic tissue: myeloperoxidase activity assay and histologic verification. J. Neurosci. Res. 29, 336-345.

Beray-Berthat, V., Croci, N., Plotkine, M. and Margaill I. (2003). Polymorphonuclear neutrophils contribute to infarction and oxidative stress in the cortex but not in the striatum after ischemia-reperfusion in rats. Brain Res. 987, 32-38.

Calapai, G., Crupi, A., Firenzuoli, F., Marciano, M. C., Squadrito, F., Inferrera, G., Parisi, A., Rizzo, A., Crisafulli, C., Fiore, A. and Caputi, A. P. (2000). Neuroprotective effects of Ginkgo billoba extract in brain ischemia are mediated by inhibition of nitric oxide synthesis. Life Sci. 67, 2673-2683.

Chopp, M., Zhang, R. L., Chen, H., Li, Y., Jiang, N. and Rusche, J. R. (1994). Postischemic administration of an anti-Mac-1 antibody reduces ischemic cell damage after transient middle cerebral artery occlusion in rats. Stroke 25, 869-875.

Chung, H. S., Harris, A., Kristinsson, J. K., Ciulla, T. A., Kagemann, C. and Ritch, R. (1999). Ginkgo biloba extract increase ocular blood flow velocity. J. Ocular Pharmacol. Ther. 15, 233-240.

DeFeudis, F. V. and Drieu, K. (2000). Ginkgo biloba extract (EGb 761) and CNS functions: basic studies and clinical applications. Curr. Drug Targets 1, 25-58.

De Lima, K. C., Schilichting, C. L., Junior, L. A., da Silva, F. M., Benetoli, A. and Milani, H. (2006). The Ginkgo biloba extract, EGb 761, fails to reduce brain infarct size in rats after transient, middle cerebral artery occlusion in conditions of unprevented, ischemia-induced fever. Phytother. Res. 20, 438-443.

De Keyser, J., Sulter, G. and Luiten P. G. (1999). Clinical trials with neuroprotective drugs in acute ischemic stroke: are we doing the right thing? Trends Neurosci. 22, 535-540.

Deters, A., Dauer, A., Schnetz, E., Fartasch, M. and Hensel, A. (2001). High molecular compounds (polysaccharides and proanthocyanidins) from Hamamelis virginiana bark: influence on human skin keratinocyte proliferation and differentiation and influence on irritated skin. Phytochemistry 58, 949-958.

Fisher, M. and Schaebitz, W. (2000). An overview of acutes stroke therapy: past, present, and future. Arch. Intern. Med. 160, 3196-3206.

Han, Y. (2005). Ginkgo terpene component has an anti-inflammatory effect on Candida albicans-caused arthritic inflammation. Int. Immunopharmacol. 5, 1049-1056.

Hwang, Y. S., Shin, C. Y., Huh, Y. and Ryu, J.H. (2002). Hwangryun-Hae-Dok-tang (Huanglian-Jie-Du-Tang) extract and its constituents reduce ischemia-reperfusion brain injury and neutrophil infiltration in rats. Life Sci. 71, 2105-2117.

Kudolo, G. E., Dorsey, S. and Blodgett, J. (2003). Effect of ingestion of Ginkgo biloba extract on platelet aggregation and urinary prostanoid excretion in healthy and type 2 diabetic 
subjects. Thromb Res. 108, 151-160.

Lee, E .J., Chen, H. Y., Wu, T. S., Chen, T. Y., Ayoub, I. A. and Maynard, K. I. (2002). Acute administration of Ginkgo biloba extract (EGb 761) affords neuroprotection against permanent and transient focal cerebral ischemia in Sprague-Dawley rats. J. Neurosci. Res. 68, 636-645.

Lenoir, M., Pedruzzi, E., Rais, S., Drieu, K. and Perianin, A. (2002). Sensitization of human neutrophil defense activities through activation of platelet-activating factor receptors by ginkgolide $\mathrm{B}$, a bioactive component of the Ginkgo biloba extract EGb 761. Biochem. Pharmacol. 63, 1241-1249.

Li, Z., Lin, X. M., Gong P. L., Du, G. H. and Zeng, F. D. (2005) Effects of gingko biloba extract on glutamate-induced $\left[\mathrm{Ca}^{2+}\right] \mathrm{i}$ changes in cultured cortical astrocytes after hypoxia/reoxyenation, $\mathrm{H}_{2} \mathrm{O}_{2}$ or L-glutamate injury. Yao Хие Xие Bao. 40, 213219.

Matsuo, T., Onodere, H., Shiga, Y., Nakamura, M., Ninomiya, M., Kihar, T. and Kogure, K. (1994). Correlation between myeloperoxidase-quantified neutrophil accumulation and ischemic brain injury in the rat. Stroke 25, 1469-1475.

Nagasawa, H. and Kogure, K. (1989). Correlation between cerebral blood flow and histologic changes in a new rat model of middle cerebral artery occlusion. Stroke 20, 1037-1043.

Oh, J. K., Hyun, S. Y., Oh, H. R., Jung, J. W., Park, C., Lee, S. Y., Park, J. H., Kim, K. H., Kim, Y. K. and Ryu, J. H. (2007). Effects of Anemarrhena asphodeloides on focal ischemic brain injury induced by middle cerebral artery occlusion in rats. Biol. Pharm. Bull. 30, 38-43.

Pardridge, W. M., Kang, Y. S., BuciaK, J. L. and Yang, J. (1995). Human insulin receptor monoclonal antibody undergoes high affinity binding to human brain capillaries in vitro and rapid transcytosis through the blood-brain barrier in vivo in the primate. Pharm. Res. 12, 807-816.
Park, Y. M., Won, J. H., Yun, K. J., Ryu, J. H., Han, Y. N., Choi, S. K. and Lee, K. T. (2006). Preventive effect of Ginkgo biloba extract (GBB) on the lipopolysaccharide-induced expressions of inducible nitric oxide synthase and cyclooxygenase- 2 via suppression of nuclear factor-kappa B in RAW 264.7 cells. Biol. Pharm. Bull. 29, 985-990.

Tanahashi, N. and Fukuuchi, Y. (2002). Treatment of acute ischemic stroke: recent progress. Intern. Med. 41, 337-344.

van Beek, T. A. (2002). Chemical analysis of Ginkgo biloba leaves and extracts. J. Chromatogr. A. 967, 21-55.

Weichel, O., Hilgert, M., Chatterjee, S. S., Lehr, M. and Klein, J. (1999). Bilobalide, a constituent of Ginkgo biloba, inhibits NMDAinduced phospholipase A2 activation and phospholipids breakdown in rat hippocampus. Naunyn Schmiedebergs Arch. Pharmacol. 360, 609-615.

Weston, R. M., Jones, N. M., Jarrott, B. and Callaway, J. K. (2007). Inflammatory cell infiltration after endothelin-1-induced cerebral ischemia: histochemical and myeloperoxidase correlation with temporal changes in brain injury. J. Cereb. Blood Flow Metab. 27, 100-114.

Yoshida, H., Yanai, H., Namiki, Y., Fukatsu-Sasaki, K., Furutani, N. and Tada, N. (2006). Neuroprotective effects of edaravone: a novel free radical scavenger in cerebrovascular injury. CNS Drug Rev. 12, 9-20.

Zhang, J., Fu, S., Liu, S., Mao, T. and Xiu, R. (2000). The therapeutic effect of Ginkgo biloba extract in SHR rats and its possible mechanism based on cerebral microvascular flow and vasomotion. Clin Hemorheol Microcirc. 23, 133-138.

Zhang, W., Sato, K., Hayashi, T., Omori, N., Nagano, I., Kato, S., Horiuchi, S. and Abe, K. (2004). Extension of ischemic therapeutic time window by a free radical scavenger, Edaravone, reperfused with tPA in rat brain. Neurol. Res. 26, 342-348. 